\title{
Strength prediction of single- and double-lap joints by standard and extended finite element modelling
}

\author{
R.D.S.G. Campilho, M.D. Banea, A.M.G. Pinto, L.F.M. da Silva, A.M.P. de Jesus
}

\author{
ABSTRACT
}

\begin{abstract}
The structural integrity of multi-component structures is usually determined by the strength and durability of their unions. Adhesive bonding is often chosen over welding, riveting and bolting, due to the reduction of stress concentrations, reduced weight penalty and easy manufacturing, amongst other issues. In the past decades, the Finite Element Method (FEM) has been used for the simulation and strength prediction of bonded structures, by strength of materials or fracture mechanics-based criteria. Cohesive-zone models (CZMs) have already proved to be an effective tool in modelling damage growth, surpassing a few limitations of the aforementioned techniques. Despite this fact, they still suffer from the restriction of damage growth only at predefined growth paths. The eXtended Finite Element Method (XFEM) is a recent improvement of the FEM, developed to allow the growth of discontinuities within bulk solids along an arbitrary path, by enriching degrees of freedom with special displacement functions, thus overcoming the main restriction of CZMs. These two techniques were tested to simulate adhesively bonded single- and double-lap joints. The comparative evaluation of the two methods showed their capabilities and/or limitations for this specific purpose.
\end{abstract}

\section{Keywords}

Bonded joint, Finite element analysis, Cohesive zone models

\section{Introduction}

The structural integrity of multi-component structures is usually determined by the strength and durability of their unions [1]. On this issue, adhesive bonding provides several advantages over welding, riveting and bolting, such as reduction of stress concentrations, reduced weight penalty and easy manufacturing [2]. Different approaches were employed in the past to predict the mechanical behaviour of bonded assemblies. In the early stages of bonded structures analyses, theoretical studies were popular [3-7], which employed simplifying assumptions in the structures geometry, materials behaviour, loading, and boundary conditions, to formulate efficient closed-form elasticity solutions for the local fields in the adhesive region. The main advantage of analytical modelling is that the structure can be analysed quickly, although with lot of embedded simplifications [8].

In the computers age, FEM codes to simulate the mechanical behaviour of structures were rapidly implemented, providing a more accurate insight on this subject. In the FEM, each component of the adhesive joint is treated as a continuum and the analysis of large displacements, such as those seen in the single-lap joints, is also available. Accounting for the materials plasticity was also made easier, since FEM codes actually incorporate several complex material laws. One of the first FEM works on bonded assemblies dates back to the 1970s when Wooley and Carver [9] conducted a stress analysis on single-lap joints. On the strength prediction of bonded assemblies, two different lines of analyses were developed over the years: the strength of materials and fracture mechanics-based methods. The strength of materials approach is based on the evaluation of allowable stresses $[10,11]$ or strains $[12,13]$, by theoretical formulations or the FEM. The assemblies strength can be predicted by comparing the respective equivalent stresses or strains at the critical regions, obtained by stress or strain-based criteria, with the properties of the structure constituents. These criteria are highly mesh dependent, as stress singularities are present at the end of the overlapping regions due to the sharp corners [14-16]. As for fracture mechanics, using LinearElastic Fracture Mechanics (LEFM), an inherent flaw is required for the calculation of the stress intensity factors or strain energy.

The limitations of the reported approaches are surpassed by CZMs, combining elements of strength and fracture approaches to derive the fracture loads $[17,18]$. The use of CZMs in fracture problems has become frequent in recent years. One of the most important advantages of CZMs is related to their ability to 
simulate onset and growth of damage without the requirement of an initial flaw, unlike classical fracture mechanics approaches. CZMs are based on spring [19] or cohesive elements [20,21], connecting plane or three-dimensional (3D) solid elements of structures. The cohesive elements should be placed along the paths where damage is prone to occur, which can be difficult to identify. However, in bonded assemblies damage growth is restricted to well defined planes, i.e., at the interfaces between the adhesive and the adherends, or cohesively in the adhesive, which allows surpassing this limitation $[22,23]$. A broad variety of works were published that prove the feasibility of this technique to model bonded assemblies, with promising results. Kafkalidis and Thouless [24] performed a FEM analysis of symmetric and asymmetric single-lap joints using a CZM approach including the adhesive plasticity by means of a traction-separation law with a trapezoidal shape. Using cohesive-zone parameters determined for the particular combination of materials used, the numerical predictions for different bonded shapes showed excellent agreement with the experimental observations. The numerical models predicted accurately the failure loads, displacements and deformations of the joints. Campilho et al. [22] evaluated the tensile behaviour of adhesively bonded single-strap repairs on laminated composites as a function of the overlap length and the patch thickness. A numerical FEM methodology including a CZM with a trapezoidal shape in pure modes I and II was used to simulate a thin ductile adhesive layer. An excellent agreement was found between the experiments and the numerical simulations on the failure modes, elastic stiffness and strength of the repairs.

The recently developed XFEM is an extension of the FEM, and its fundamental features were presented in first hand in the late 1990s by Belytschko and Black [25]. It is based on the idea of partition of unity presented by Melenk and Babuska [26], which consists on local enrichment functions for the nodal displacements to model crack growth and separation between crack faces [27]. With this technique, discontinuities such as cracks are simulated as enriched features, by allowing discontinuities to grow through the enrichment of the degrees of freedom of the nearby nodes with special displacement functions. As the cracktip changes its position and path due to loading conditions, the XFEM algorithm creates the necessary enrichment functions for the nodal points of the finite elements around the crack path/tip. Compared to CZMs, XFEM excels in simulating crack onset and growth along an arbitrary path without the requirement of the mesh to match the geometry of the discontinuities neither remeshing near the crack [28]. This can be an advantage to CZM modelling for the simulation of bonded engineering plastics or polymer-matrix composites, where adherend cracking may occur after initiation in the adhesive.

Varying applications to this innovative technique were proposed to simulate different engineering problems. In 2000, Sukumar et al. [29] updated the method to three-dimensional damage simulation. Modelling of intersecting cracks with multiple branches, multiple holes and cracks emanating from holes were addressed by Daux et al. [30]. The problem of cohesive propagation of cracks in concrete structures was studied by Moës and Belytschko [31], considering three-point bending and four-point shear scaled specimens. More advanced features such as plasticity, contacting between bodies and geometrical nonlinearities, which show a particular relevance for the simulation of fracture in structures, are already available within the scope of XFEM. The employment of plastic enrichments in XFEM modelling is accredited to Elguedj et al. [32], which used a new enriched basis function to capture the singular fields in elasto-plastic fracture mechanics. Modelling of contact by the XFEM was firstly introduced by Dolbow et al. [33] and afterwards adapted to frictional contact by Khoei and Nikbakht [34]. Fagerström and
Larsson [35] implemented geometrically non-linearities within XFEM.

This work aims the comparison and evaluation of CZM and XFEM modelling, currently implemented in the FEM package ABAQUS $^{s}$, to simulate the behaviour of adhesively bonded singleand double-lap joints between aluminium adherends, bonded with the brittle adhesive Araldite ${ }^{\mathbf{s}}$ AV138. The study comprises a variety of overlap lengths, between 5 and $20 \mathrm{~mm}$, to test both modelling solutions under different conditions, between an approximately even level of shear stresses along the bond up to the large shear stress gradients found in joints with bigger bond lengths. This work will equally allow the discussion of the capabilities and/or limitations of these two methods to model bonded structures, by direct comparisons with experimental data.

\section{Experimental work}

\subsection{Characterisation of the materials}

The aluminium alloy AW6082 T651 was selected for the adherends, characterised by a high tensile strength (340 MPa as specified by the manufacturer) obtained through artificial ageing at a temperature of approximately $1801 \mathrm{C}$ [36]. This specific alloy was chosen due to its wide use in Europe for several structural applications under different extruded and laminated shapes. The bulk stress-strain (s-e) response of the aluminium adherends, obtained according to the standard ASTM-E8M-04 [37], is presented in Fig. 1 for the three specimens tested. The aluminium has a Young's modulus of $70.0770 .83 \mathrm{GPa}$, a yield stress of 261.677 $7.65 \mathrm{MPa}$, a maximum strength of $32470.16 \mathrm{MPa}$ and a failure strain of $21.7074 .24 \%$. The bilinear approximation of Fig. 1 was used as input in the simulations. The adhesive Araldite ${ }^{\mathbf{s}}$ AV138 was also characterised for input in the FEM analysis. The characterisation tests for the adhesive were carried out under tension (mode I loading) and shear (mode II loading) considering three specimens for each condition, which allowed the determination of the yield strengths and moduli in both loadings. The adhesive bulk specimens for mode I loading were fabricated according to the French standard NF T 76-142 [38] to assume porosity-free specimens. Thus, the specimens were made of $2 \mathrm{~mm}$ plates fabricated in a sealed mould, followed by machining to produce the dogbone shape described in the standard. The Thick Adherend Shear Test (TAST) tests for mode II loading followed the guidelines of the standard ISO 11003-2:1999 [39], using DIN Ck 45 steel for the adherends. Particular attention was paid to the surface preparation and bonding procedures to guarantee cohesive failures of the adhesive, which followed entirely the indications of the standard. Fig. 2 shows typical stress-strain curves in pure mode I of the

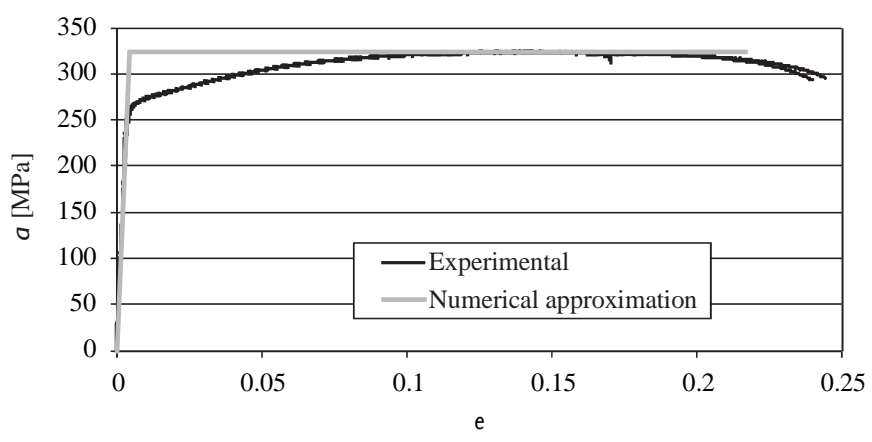

Fig. 1. Experimental S-e curves of the aluminium AW6082 T651 and approximation for the FEM analysis. 


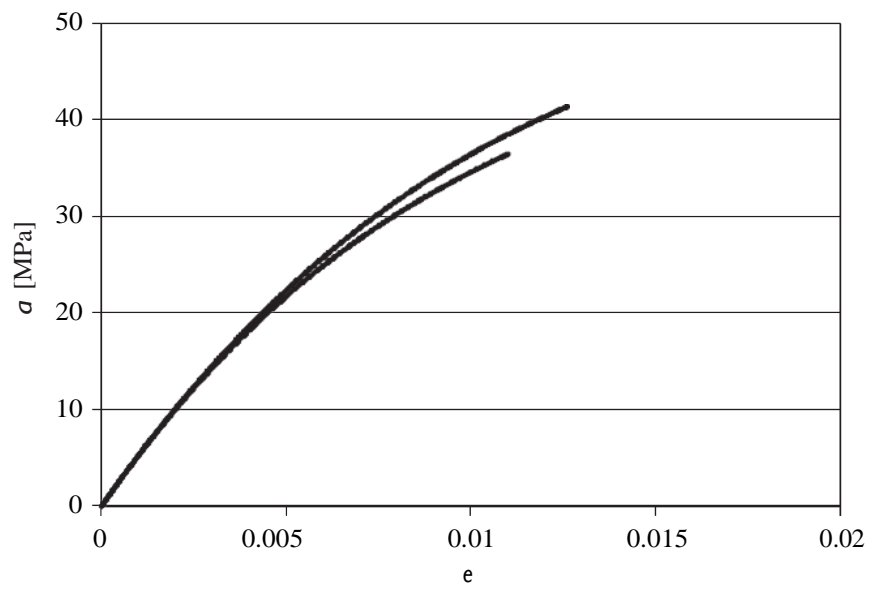

Fig. 2. Experimental S-e curves of the Araldite ${ }^{\text {S }}$ AV138.

Table 1

Properties of the adhesive Araldites AV138 [40].

\begin{tabular}{ll}
\hline Property & AV138 \\
\hline Young's modulus, $E(\mathrm{GPa})$ & 4.8970 .81 \\
Poisson's ratio, $\mathrm{n}^{\mathrm{a}}$ & 0.35 \\
Tensile yield strength, $\mathrm{S}_{y}(\mathrm{MPa})$ & 36.4972 .47 \\
Tensile failure strength, $\mathrm{S}_{f}(\mathrm{MPa})$ & 39.4573 .18 \\
Tensile failure strain, $\mathrm{e}_{f}(\%)$ & 1.2170 .10 \\
Shear modulus, $G(\mathrm{GPa})$ & 1.5670 .01 \\
Shearyield strength, $\mathrm{t}_{y}(\mathrm{MPa})$ & 25.170 .33 \\
Shear failure strength, $\mathrm{t}_{f}(\mathrm{MPa})$ & 30.270 .40 \\
Shear failure strain, $\mathrm{g}_{f}(\%)$ & 7.870 .7 \\
\hline
\end{tabular}

${ }^{a}$ Manufacturer's data.

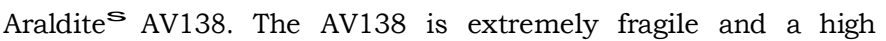
deviation was found since, due to its brittleness, it is highly sensitive to fabrication defects [40]. The yield strength was calculated for a plastic deformation of $0.2 \%$. Details about the TAST tests can be found in Ref. [40]. Table 1 summarises the collected data on these materials, which will be subsequently used for the finite element simulations and strength predictions [40].

\subsection{Joint geometries}

The geometry and dimensions of the single- and double-lap joints are detailed in Fig. 3. The following values were selected for this work: plate thickness $t_{P} 1 / 43 \mathrm{~mm}$, adhesive thickness $t_{A} 1 / 40.2 \mathrm{~mm}$, overlap length $L_{O} 1 / 45,10$ and $20 \mathrm{~mm}$, and joint total length between grips $L_{T}^{1 / 4} 180 \mathrm{~mm}$. Aluminium tabs were glued at the specimens edges for a correct alignment in the testing machine. For the fabrication of the specimens, the adherends were initially cut from a bulk plate and then machined to the final dimensions. The bonding surfaces were grit blasted and cleaned with acetone before bonding, which was performed using an apparatus for the correct alignment. Fishing lines with a calibrated diameter of $0.2 \mathrm{~mm}$ were inserted between the adherends at the overlap edges to assure the correct value of $t_{A}$. The correct alignment and positioning of the adherends to produce the different values of $L_{O}$ was performed with a digital calliper. Curing of the specimens was carried out according to the manufacturer's specifications for complete curing, i.e., for at least $48 \mathrm{~h}$ at room temperature. The tests were carried out in a Shimadzu AG-X 100 testing machine with a $100 \mathrm{kN}$ load cell, at room temperature and under displacement control $(2 \mathrm{~mm} / \mathrm{min})$. Four valid results were
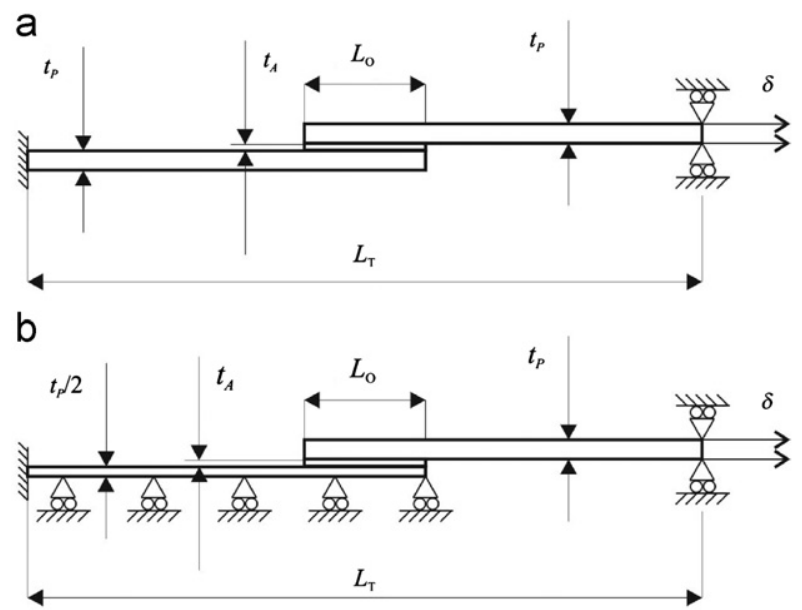

Fig. 3. Geometry and dimensions of the single-lap joint (a) and double-lap joint (b).

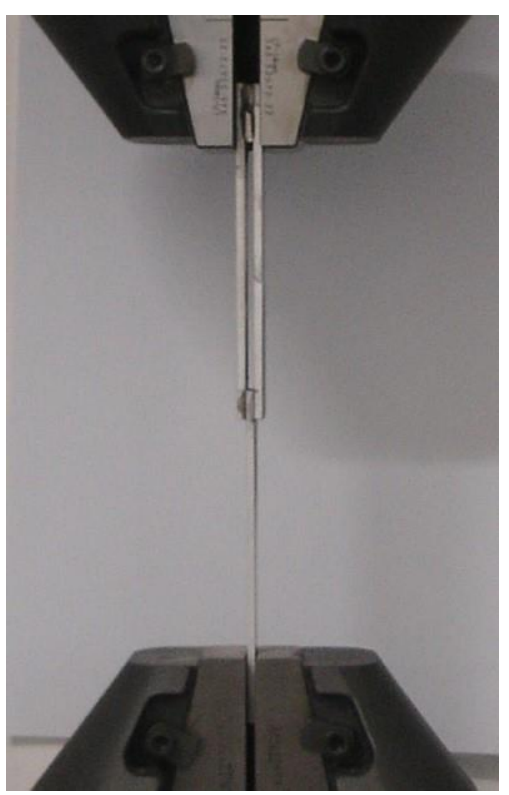

Fig. 4. Double-lap joint with $L_{O}^{1 / 4} 10 \mathrm{~mm}$ in the testing machine under testing.

always provided for each condition. Fig. 4 shows a double-lap joint with $L_{O} 1 / 410 \mathrm{~mm}$ in the testing machine under testing.

\section{Numerical analysis}

A numerical analysis was performed in the commercial FEM package ABAQUS ${ }^{S}$ to assess the viability of its CZM and XFEM embedded formulations, already discussed in terms of generic principles, in predicting the strength of adhesively bonded singleand double-lap joints. The numerical analysis was carried out using non-linear geometrical considerations with the material properties and simplified elastic-plastic laws depicted in Section 2. The FEM meshes were built without symmetry conditions for the single-lap joints (Fig. 3(a)) and with horizontal symmetry for the double-lap joints (Fig. 3(b)), to reduce the total number of elements. Fig. 5 shows two representative meshes of the CZM and XFEM damage modelling analyses, considering the standard refinement that was used for this study. Restraining and loading conditions were introduced to faithfully model the real testing conditions, consisting on clamping of the joint at one edge and 
a

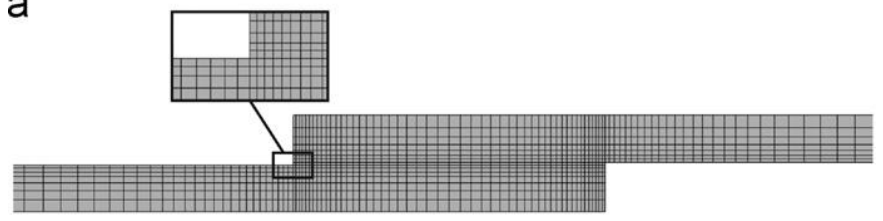

b

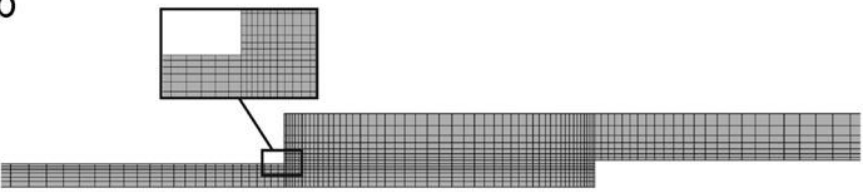

Fig. 5. FEM meshes for the single-lap joint (a) and double-lap joint (b) with $L_{O} 1 / 420 \mathrm{~mm}$; CZM modelling.

applying a vertical restraint and tensile displacement at the opposite edge $[41,42]$. The meshes were constructed taking advantage of the automatic meshing algorithms of ABAQUS ${ }^{\text {, from a }}$ manual seeding procedure that included biasing towards the overlap edges, since these theoretically singular regions show large stress gradients, thus allowing to accurately capture these phenomena $[2,18]$. The joints were modelled as two-dimensional, with plane-strain solid elements (referenced as CPE8 from the ABAQUS ${ }^{5}$ library). While for the CZM analysis, the adhesive was modelled by a traction-separation law including the adhesive layer stiffness, as detailed in Section 3.1, for the XFEM model, the adhesive layer was modelled by the same elements used for the adherends, considering one layer of solid elements. Both of the techniques that will be employed for the simulation of damage are currently implemented within ABAQUS ${ }^{s}$ CAE suite and will be briefly described in the following.

\subsection{Cohesive zone modelling}

CZMs model the elastic loading, initiation of damage and further propagation due to local failure within a material. CZMs are based on a relationship between stresses and relative displacements connecting initially superimposed nodes of the cohesive elements (Fig. 6), to simulate the elastic behaviour up to a peak load and subsequent softening, to model the gradual degradation of material properties up to complete failure. Generically speaking, the shape of the softening laws can be adjusted to conform to the behaviour of the material or interface they are simulating $[22,23]$. The areas under the traction-separation laws in each mode of loading (tension and shear) are equalled to the respective fracture energy. Under pure mode, damage propagation occurs at a specific integration point when the stresses are released in the respective traction-separation law. Under mixed mode, energetic criterions are often used to combine tension and shear [22], thus simulating the typical mixed mode behaviour inherent to bonded assemblies. In this work, a continuum-based approach, i.e. using the cohesive elements to model solids rather interfaces, was considered to model the finite thickness of the adhesive layer. The cohesive layer is assumed to be under one direct component of strain (through-thickness) and one transverse shear strain, which are computed directly from the element

kinematics. The membrane strains are assumed as zero, which is appropriate for thin and compliant layers between stiff adherends. The strength predictions of CZM modelling are expected to be mesh independent. A study is carried out further in this work (Section 4.2) to evaluate this issue.

The traction-separation law assumes an initial linear elastic behaviour followed by linear evolution of damage. Elasticity is

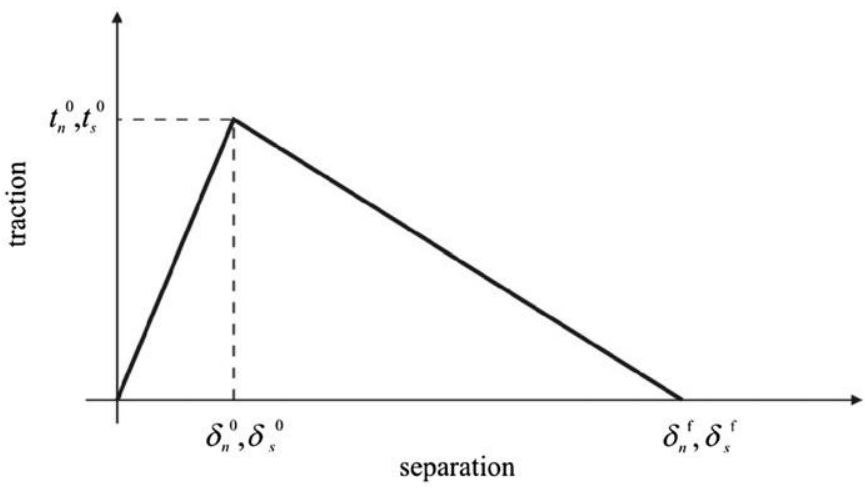

Fig. 6. Traction-separation law with linear softening available in ABAQUS $\mathbf{s}$.

Table 2

Properties of the adhesive Araldite ${ }^{\mathbf{s}}$ AV138 for CZM modelling [40].

\begin{tabular}{lclc}
\hline$E(\mathrm{GPa})$ & 4.89 & $G(\mathrm{GPa})$ & 1.56 \\
$t_{n}^{(\mathrm{MPa})}$ & 39.45 & $t 0(\mathrm{MPa})$ & 30.2 \\
$G_{n}^{c}(\mathrm{~N} / \mathrm{mm})$ & 0.2 & $G_{s}^{s}(\mathrm{~N} / \mathrm{mm})$ & 0.38 \\
$n_{n}$ & & ${ }_{s}$ & \\
\hline
\end{tabular}

defined by an elastic constitutive matrix relating stresses and strains across the interface [43]

The $\mathbf{t}=\left\{\begin{array}{l}t_{n} \\ t_{s}\end{array}\right\}=\left[\begin{array}{ll}K_{n n} & K_{n s} \\ K_{n s} & K_{s s}\end{array}\right] \cdot\left\{\begin{array}{l}E_{n} \\ \varepsilon_{s}\end{array}\right\}=\mathbf{K} \varepsilon$

layer, given by the relevant elastic modul1. A suitable approximation for thin adhesive layers is provided with $K_{n n} 1 / 4 E, K_{s s}^{1 / 4} G, K_{n s}$ $1 / 40 ; E$ and $G$ are the longitudinal and transverse elastic moduli, respectively [22]. Damage initiation can be specified by different criteria. In this work, the quadratic nominal stress criterion was considered for the initiation of damage, already shown to give accurate results [23], expressed as [43]

$$
\left\{\frac{\left\langle t_{n}\right\rangle}{t_{n}^{0}}\right\}^{2}+\left\{\frac{t_{s}}{t_{s}^{0}}\right\}^{2}=1
$$

$t_{n}^{0}$ and $t_{s}^{0}$ represent the pure mode (normal or shear, respectively) peakvalues of the nominalstress. /S are the Macaulay brackets, emphasising that a purely compressive stress state does not initiate damage. After the peak value in Fig. 6 is attained, the material stiffness is degraded under different possible laws, depending on the material to be simulated. For brittle materials such as the Araldite ${ }^{\mathbf{S}}$ AV138, a linear softening law is sufficiently appropriate, Fig. 6 [44]. Complete separation is predicted by a linear power law form of the required energies for failure in the pure modes [43]

$$
\frac{G_{m}}{G_{n}}+\frac{G_{s}}{G_{s}^{c_{s}}}=1
$$

The quantities $G_{n}$ and $G_{s}$ relate to the work done by the traction and corresponding relative displacements in the normal and shear directions, whilst the relating critical fracture energies required for pure mode failure are given by $G_{n}^{c}$ and $G_{s}^{c}$ for normal and shear loadings, respectively. Table 2 shows the values introduced in ABAQUS ${ }^{s}$ for the simulation of damage growth in the adhesive layer [40]. These properties were estimated from the data of Table 1, considering the average values of failure strength from the characterisation tests to define $t$ and $t^{\circ}$, and considering typical values for brittle adhesives for ${ }^{n} G^{c}$ and $G^{c}$, followed by fitting of these two parameters for one of the testing 
configurations (single-lap joint with $L_{O} 1 / 420 \mathrm{~mm}$ ). These values were subsequently applied to all configurations tested.

\section{2. eXtended Finite Element Modelling}

The XFEM is also tested in this work to assess its feasibility in simulating damage propagation in adhesively bonded joints. The XFEM formulation embedded in ABAQUS ${ }^{s}$ CAE suite was used, whose basic principles and analysis technique are briefly described in this section [43]. As an extension to the conventional FEM, the XFEM is based on the integration of enrichment functions in the Finite Element formulation, although retaining its basic properties such as sparsity and symmetry of the resulting stiffness matrix. These functions allow modelling the displacement jump between crack faces that occur during the propagation of a crack. The fundamental expression of the displacement vector $\mathrm{u}$, including the displacements enrichment, is written as [43]

$$
\mathbf{u}=\sum_{i=1}^{N} N_{i}(x)\left[\mathbf{u}_{i}+H(x) \mathbf{a}_{i}+\sum_{\alpha=1}^{4} F_{\alpha}(x) \mathbf{b}_{i}^{\alpha}\right]
$$

$N_{i}(x)$ and $\mathrm{u}_{i}$ relate to the conventional FEM technique, corresponding to the nodal shape functions and nodal displacement vector linked to the continuous part of the formulation, respectively. The second term between brackets, $H(x) \mathrm{a}_{i}$, is only active in the nodes for which any relating shape function is cut by the crack and can be expressed by the product of the nodal enriched degree of freedom vector including the mentioned nodes, $\mathrm{a}_{i}$, with the associated discontinuous shape function, $H(x)$, across the crack surfaces

$$
H(x)= \begin{cases}1 & \text { if }\left(\mathbf{x}-\mathbf{x}^{*}\right) \cdot \mathbf{n} \geq 0 \\ -1 & \text { otherwise }\end{cases}
$$

$\mathrm{x}$ is a sample Gauss integration point, $\mathrm{x}^{*}$ is the point of the crack closest to $\mathrm{x}$, and $\mathrm{n}$ is the unit vector normal to the crack at $\mathrm{x}^{*}$ (Fig. 7). Finally, the third term is only to be considered in nodes whose shape function support is cut by the crack tip and is given by the product of the nodal enriched degree of freedom vector of this set of nodes, $\mathrm{b}_{i}^{\mathrm{a}}$, and the associated elastic asymptotic crack-tip functions, $F_{\mathrm{a}}(x)$ [45]. $F_{\mathrm{a}}(x)$ are only used in ABAQUS ${ }^{s}$ for stationary cracks, which is not the current scenario. In the presence of damage propagation, a different approach is undertaken, based on the establishment of phantom nodes that subdivide elements cut by a crack and simulate separation between the newly created subelements. By this approach, the asymptotic functions are discarded, and only the displacement jump is included in the formulation. Propagation of a crack along an arbitrary path is made possible by the use of phantom nodes that initially have exactly the same coordinates than the real nodes and that are completely constrained to the real nodes up to damage initiation. In Fig. 8, thehighlighted element has nodes $n_{1}$ to $n_{4}$. After being crossed by a crack at! , the element is partitioned in two sub-domains, $\mathrm{O}_{\mathrm{A}}$ and $\mathrm{O}_{\mathrm{B}}$. The discontinuity in the displacements is made possible by adding phantom nodes ( $\tilde{n}_{1}$ to $\tilde{n_{4}}$ ) superimposed to the original nodes. When an element cracks, each one of the two sub-elements will be formed by real nodes (the ones corresponding to the cracked part) and phantom nodes (the ones that no longer belong to the respective part of the original element). These two elements that have fully independent displacement fields replace the original one, constituted by the nodes $\tilde{n}_{1}, \tilde{n}_{2}, n_{3}$ and $n_{4}\left(\mathrm{O}_{\mathrm{A}}\right)$ and $n_{1}, n_{2}, \tilde{n}_{3}$ and $\tilde{n_{4}}$ $\left(\mathrm{O}_{\mathrm{B}}\right)$. From this point, each pair of real/phantom node of the cracked element is allowed to separate according to a suitable cohesive law up to failure. At this stage, the real and phantom nodes are free to move unconstrained, simulating crack growth. In terms of damage initiation, ABAQUS ${ }^{5}$ allows the user to define initial cracks, but this is not mandatory. Regardless the choice taken, ABAQUS ${ }^{5}$ initiates and propagates damage during the simulation at regions experiencing principal stresses and/or strains greater than the corresponding

initiation/propagation will always take place orthogonally to the maximum principal stresses or strains. By the described principles, it is supposed that any strength prediction data is relatively mesh independent since crack growth is ruled by energetic criteria. Oppositely, if the prediction of failure is carried out by the damage initiation criteria, some variations are expected, as stresses/strains at concentration regions are mesh dependent. A study is performed in Section 4.3 for clarification. Table 3 summarises the parameters

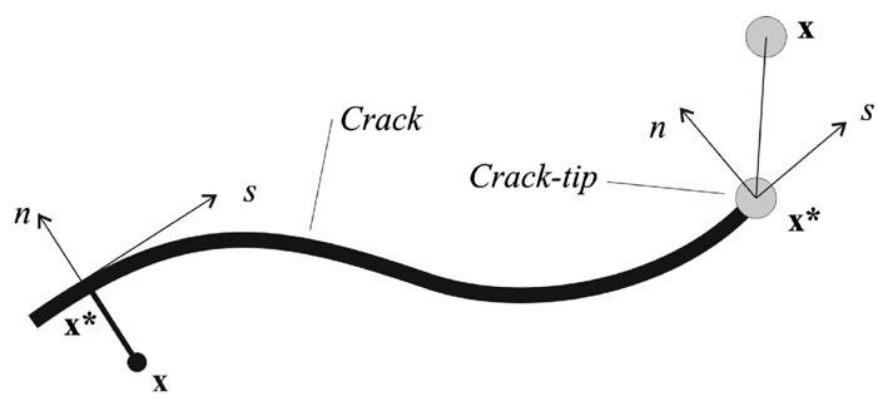

Fig. 7. Representation of normal and tangential coordinates for an arbitrary crack.
Table 3

Properties of the adhesive Araldite ${ }^{\mathbf{s}}$ AV138 and aluminium alloy AW6082 T651 for XFEM modelling.

\begin{tabular}{lll}
\hline & $\begin{array}{l}\text { Adhesive Araldite } \\
\text { AV138 }\end{array}$ & $\begin{array}{l}\text { Aluminium } \\
\text { AW6082 T651 }\end{array}$ \\
\hline$E(\mathrm{GPa})$ & 4.89 & 70.07 \\
$G(\mathrm{GPa})$ & 1.56 & 26.34 \\
$S_{n}^{0}(\%)$ & 1.21 & 21.70 \\
$G_{n}^{c}(\mathrm{~N} / \mathrm{mm})$ & 0.2 & $15^{\mathrm{a}}$ \\
$G_{s}^{c}(\mathrm{~N} / \mathrm{mm})$ & 0.38 & $15^{\mathrm{a}}$ \\
\hline
\end{tabular}

${ }^{a}$ Merely indicative values from the literature. a

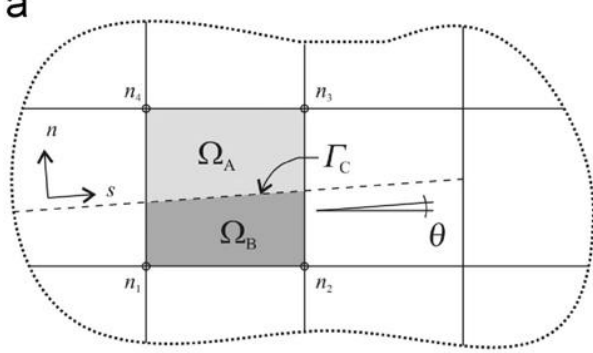

b

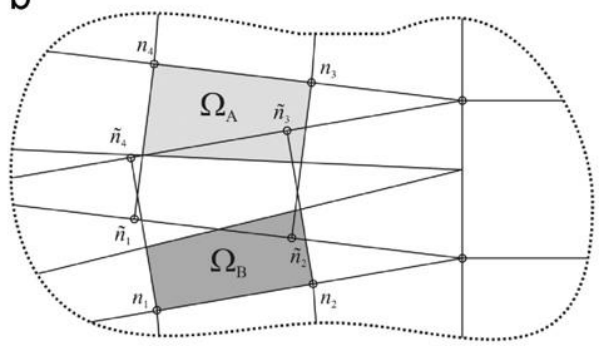

Fig. 8. Damage propagation in XFEM using the phantom nodes concept: before (a) and after partitioning (b) of a cracked element into sub-elements. 
introduced in ABAQUS $^{s}$ for damage propagation in the adhesive layer and aluminium adherends. $\S^{0}$ represents the maximum principal strain that will lead to damage initiation. It should be emphasised that, due to the intrinsic principles of XFEM as explained above, only one strength/strain parameter is to be introduced in ABAQUS ${ }^{\mathbf{s}}$, corresponding to the maximum principal strength/strain that will trigger the initiation of damage. In Table 3 , the value of $\AA^{0}$ for the aluminium adherends is defined from the average value of failure strain obtained in the tensile bulk tests to this material. The values of $G_{n}^{c}$ and $G^{c}$ are typical values from the aluminium literature.

\section{Results and discussion}

\subsection{Fracture modes}

The experiments revealed for all joints a cohesive failure of the adhesive bond, which testifies the effectiveness of the chosen adhesive and surface preparation method to bond the aluminium

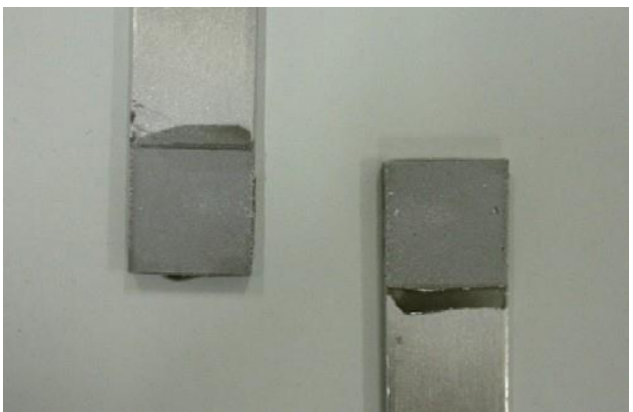

Fig. 9. Cohesive fracture surface, representative of the failure mechanism of all joints tested, for a $L_{O} 1 / 420 \mathrm{~mm}$ single-lap joint. adherends. Fig. 9(a) shows a cohesive failure of the adhesive layer for a $L_{O} 1 / 420 \mathrm{~mm}$ single-lap joint.

\subsection{CZM modelling}

In the simulations, by modelling the adhesive layer as a traction-separation law with CZMs and the adherends as elastic-perfectly plastic using the approximation of Fig. 1, fracture occurred due to cohesive crack propagation in the adhesive bond, beginning at the overlap edges with fast propagation to the

inner regions of the bond. Fig. 10 shows the failure process of the $L_{O} 1 / 420 \mathrm{~mm}$ single-lap joint, representative of the full range of geometries considered (the parameter SDEG corresponds to the stiffness degradation, with SDEG $1 / 40$ relating to the undamaged material and SDEG $1 / 41$ to complete failure). Damage initiated cohesively in the adhesive layer at the overlap edges, propagating towards the inner region of the bond up to complete failure. All load-displacement $(P-\mathrm{d})$ curves were typically linear up to failure, and no plastic deformation of the adherends was found neither in the tests nor in the simulations, mainly due to the high strength of the aluminium. Additionally, the adhesive Araldite $^{\text {s }}$ AV138 is an extremely brittle adhesive $[46,47]$, as testified in the bulk tensile tests showed in Fig. 2. Fig. 11 depicts the experimental and numerical (CZM modelling) $P-\mathrm{d}$ curves for the single-lap joints with $L_{O} 1 / 45 \mathrm{~mm}$ (a) and $L_{O} 1 / 420 \mathrm{~mm}$ (b). Fig. 12 shows the $P-\mathrm{d}$ curves for the double-lap joints with equivalent dimensions, i.e., $L_{O} 1 / 45 \mathrm{~mm}$ (a) and $L_{O} 1 / 420 \mathrm{~mm}$ (b). The non-linear behaviour of the experimental $P$-d curves of Fig. 12(b) initiating at $\mathbf{E} 0.7 \mathrm{~mm}$, not visible in the numerical prediction, is related to the onset of yielding (Fig. 1), which is not considered in the elastic-perfectly plastic numerical approximation. The comparative analysis between the tests and simulations shows the suitability of CZM modelling in capturing all the relevant features of the failure process of these joints, such as the value of a

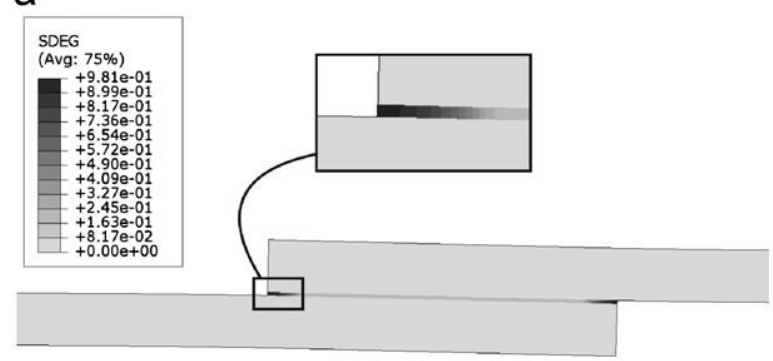

b

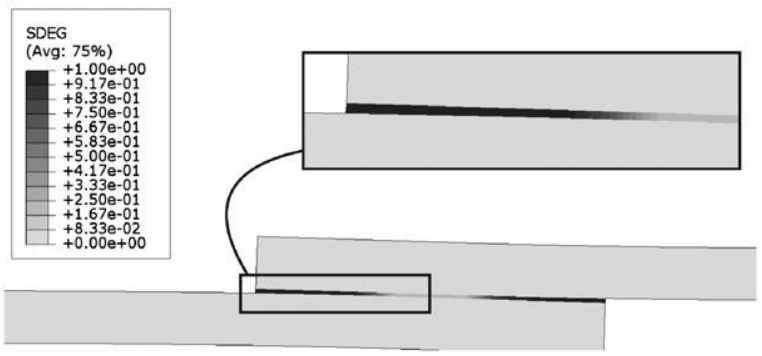

Fig. 10. Progressive failure in the adhesive layer for a $L_{O} 1 / 420 \mathrm{~mm}$ single-lap joint using CZMs; damage initiation at the overlap edges (a) and propagation to the inner region of the bond (b); SDEG corresponds to the stiffness degradation, with SDEG 1/4 0 relating to the undamaged material and SDEG $1 / 41$ to complete failure.

a

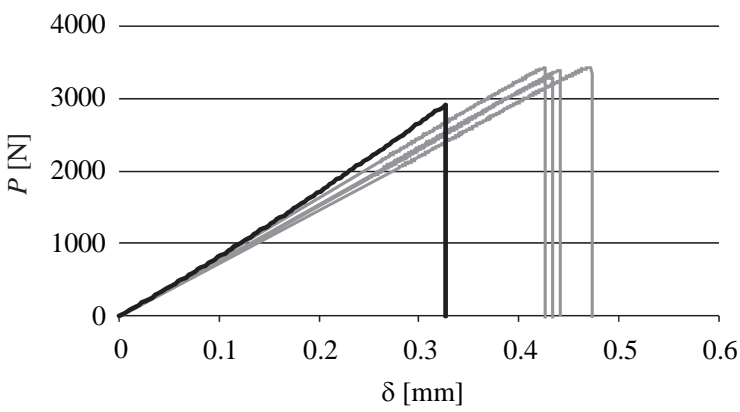

-Experimental —Numerical b

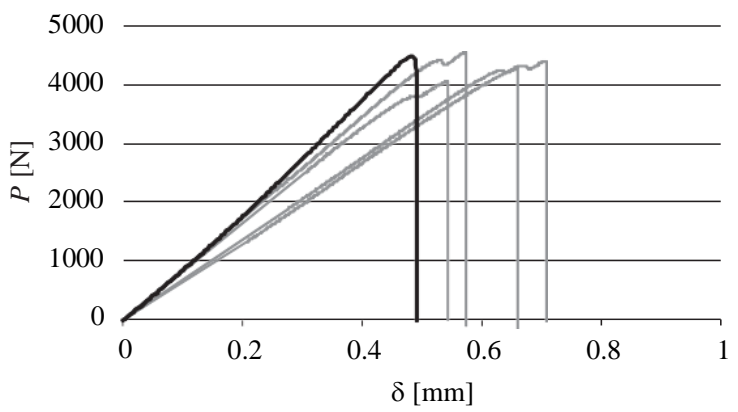

- Experimental —Numerical

Fig. 11. Experimental and numerical (CZM) $P$-d curves for the single-lap joints with $L_{O} 1 / 45 \mathrm{~mm}$ (a) and $L_{O} 1 / 420 \mathrm{~mm}$ (b). 
a

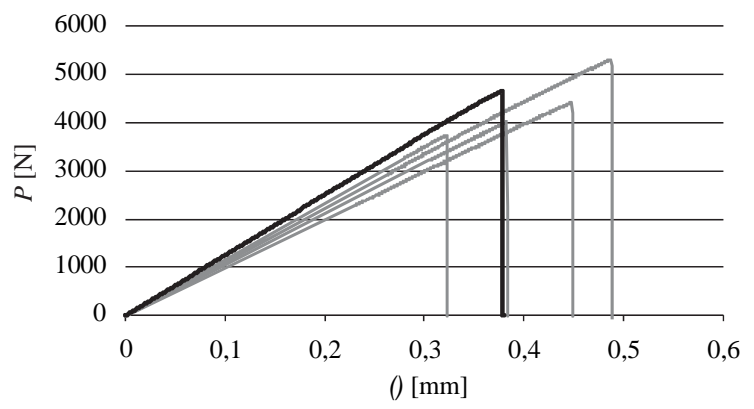

Experimental b

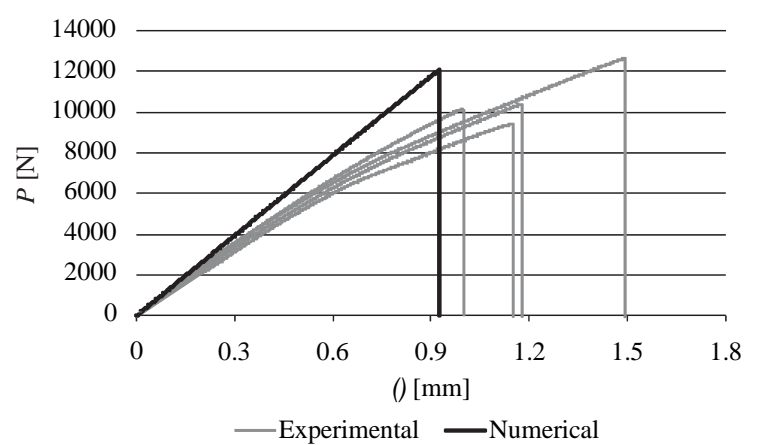

Fig. 12. Experimental and numerical (CZM) $P$-d curves for the double-lap joints with $L_{O} 1 / 45 \mathrm{~mm}$ (a) and $L_{O} 1 / 420 \mathrm{~mm}$ (b).

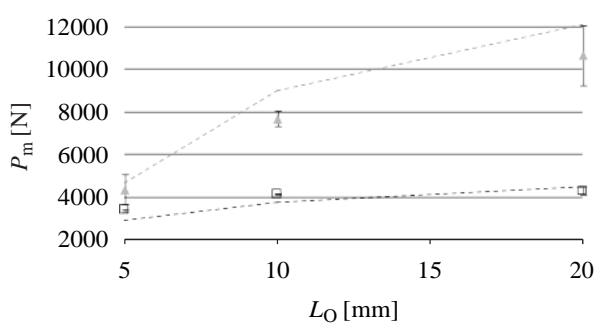

口 Single-lap Experimental -..-- Single-lap Numerical (CZM)

$\triangle$ Double-lap Experimental …- Double-lap Numerical (CZM) b

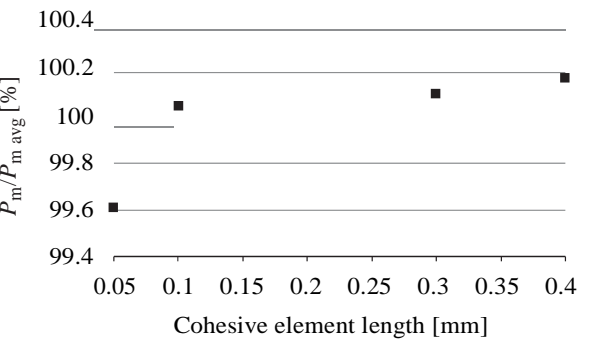

Fig. 13. Experimental and numerical (CZM modelling) values of $P_{m}$ as a function of $L_{O}(\mathrm{a})$ and mesh dependency study for the $L_{O}{ }^{1 / 4} 20$ mm single-lap joint $\quad$ (b).

maximum load sustained by the specimens $\left(P_{m}\right)$, stiffness or failure displacement $[22,24]$. As a validation of CZM modelling for the simulation of adhesively bonded joints, the value of $P_{m}$ is plotted against the experimental data in Fig. 13(a). All of the test data includes the average value for each quantity and deviation of the four tested specimens. The results were quite close, with the biggest difference (E 17\%) being found for the $L_{O} 1 / 410 \mathrm{~mm}$ double-lap joint. A mesh dependency study was also performed (Fig. 13(b)), to evaluate the influence of the mesh refinement for the cohesive elements representative of the adhesive layer in the global results. The $L_{O} 1 / 420 \mathrm{~mm}$ single-lap joint was tested with this purpose, as it provides the largest gradient of stresses at the adhesive bond. In all the simulations bias effects were considered towards the overlap edges, with average element lengths at these regions between 0.05 and $0.4 \mathrm{~mm} . P_{m} / P_{\text {mavg }}$ refers to the joint strength normalised to the average strength between all element sizes. Fig. 13(b) testifies the small influence of the mesh size at the adhesive bond, by showing values of $P_{m} / P_{\text {mavg }}$ between approximately $99.6 \%$ and $100.2 \%$. This behaviour is characteristic of CZM modelling [48] since an energetic criterion, based on the fracture toughness of the material, is used for the damage growth. Since the energy required for propagation is averaged over the damaged area, opposed to the use of a discrete value of maximum stress/strain as it happens for the strength of materials criteria, results are mesh independent provided that a minimum refinement is used $[22,24]$.

\subsection{XFEM modelling}

Using XFEM modelling for the propagation of damage, different properties had to be set for damage in the adhesive layer and in the aluminium adherends, imposed based on the experimental tests reported on Section 2 (Table 3). It should be pointed out that the current implementation of the XFEM in ABAQUS ${ }^{s}$ is restricted to only one value of maximum strength or strain leading to the initiation of damage (by the maximum principal stress or strain criterion, respectively), which can be a severe limitation since the fracture process of thin adhesive layers is not consistent with that of bulk materials, due to the constraining effects imposed by the surrounding stiff adherends [49]. This does not allow the separation of the adhesive behaviour into tensile and shear behaviour that is performed for cohesive zones models, which can be in some cases mandatory for the accuracy of the results if large constraining effects are present in the bond [23]. Apart from this feature, the current implementation of the method itself involves an even more important handicap. It is known that, if no initial cracks are introduced in the models, the XFEM algorithm will automatically search for the maximum principal stresses/strains in each one of the structure materials (in the present scenario, in both the adhesive and adherends), to initiate damage propagation in the first locus in which these stresses/strains surpass the respective material properties. During damage propagation, the XFEM algorithm continuously searches for the principal stress/strain direction at the crack tip, to specify the direction of subsequent crack growth $[27,31]$. For the specific case of singlelap or double-lap joints, cracking initiates in the adhesive bond orthogonally to the direction of principal stresses/stresses, growing up to the adhesive/aluminium interface. Fig. 14(a) shows this process for a $L_{O} 1 / 420 \mathrm{~mm}$ single-lap joint (detail at the overlap edge) using the principal strain criterion for the initiation of damage and direction of crack growth. At this point, the direction of maximum strain leads to propagation of damage towards the aluminium adherend. When the crack reaches the interface, damage will propagate almost vertically due to the new direction of principal strains at the crack tip (Fig. 14(b)), which clearly does 
a

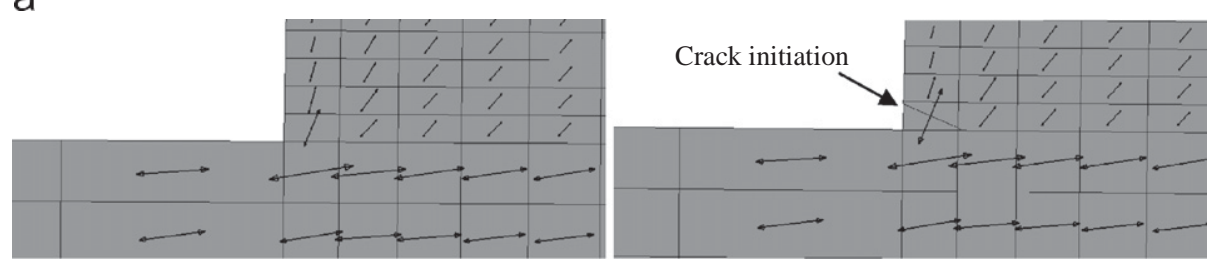

b

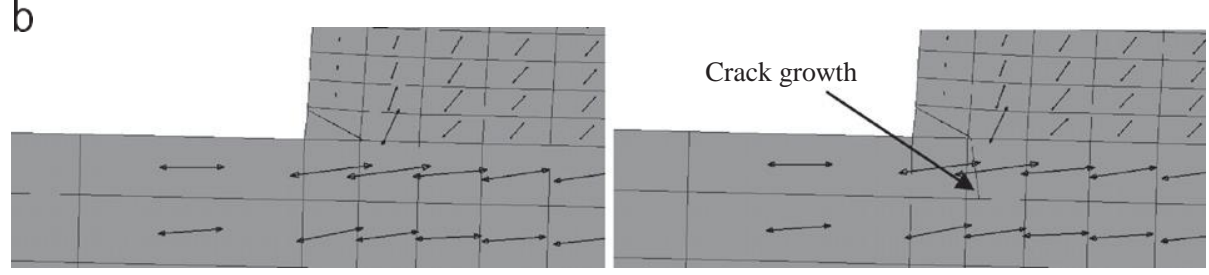

Fig. 14. Progressive failure of a $L_{O} 1 / 420 \mathrm{~mm}$ single-lap joint using XFEM (the arrows represent the directions of maximum principal strain): damage initiation within the adhesive at the overlap edges (a) and damage growth to the aluminium adherend (b).

a

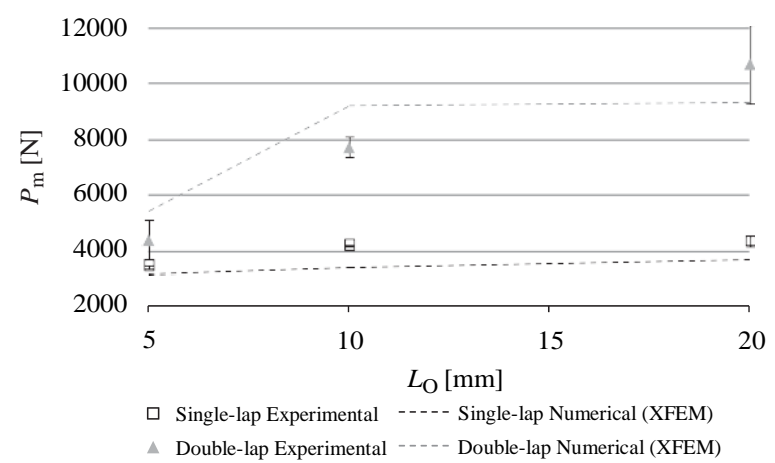

b

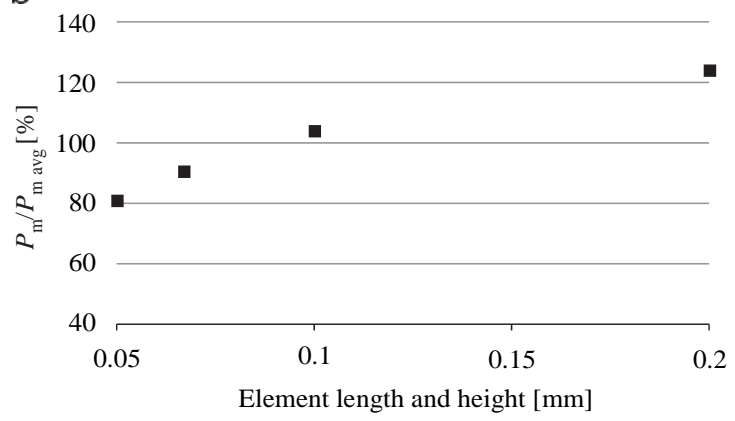

Fig. 15. Experimental and numerical (XFEM modelling) values of $P_{m}$ as a function of $L_{O}(\mathrm{a})$ and mesh dependency study for the $L_{O}{ }^{1 / 4} 20 \mathrm{~mm}$ single-lap joint $\quad$ (b).

not reflect the real behaviour of single-lap joints. Damage propagation along the adhesive bond is thus rendered unfeasible with this technique, since the algorithm will always search for maximum stresses/strains at the crack tip, shifting the crack to the adherends, disregarding what happens within the adhesive layer and thus preventing damage propagation along the adhesive bond. From this discussion it becomes clear that XFEM, as it is currently implemented, is only suitable for the identification of the locus of damage initiation in adhesive bonds, by comparing the maximum principal stress/strain in each of the constituent materials to the respective maximum values. However, it does not show to be suited for the simulation of damage growth, as the principle for defining the crack direction (orthogonal to the maximum principal stress/strain) does not model accurately the propagation of damage in multi-material structures as it does not consider the initiation of damage outside the tip of the cracks that emerge from the structure boundaries nor does it take into account the prospect of damage growth along interfaces between different materials. For the specific case of bonded joints, a modification of the XFEM algorithm that would consider these possibilities would bring a significant breakthrough for the simulation of these structures, with the accuracy of CZMs but eliminating the major handicap of this method to follow the damage paths specified by the placement of the cohesive elements. As a result of this handicap, a different solution is proposed, supported by the brittleness of the adhesive used. The maximum strength of the joints will be predicted by the initiation of cohesive cracking of the adhesive layer at the overlap edges, using the maximum principal strain criterion as it showed to be slightly less mesh sensitive than the maximum principal stress criterion. Fig. 15(a) compares the experimental and XFEM data considering the maximum principal strain criterion, showing that the XFEM is moderately accurate in simulating these structures with brittle adhesives that lead to a catastrophic failure of the joint as soon as the maximum strain of the adhesive is attained anywhere in the structure. However, the proposed methodology was only acceptable due to the brittleness of the adhesive since, if a ductile adhesive had been used instead, the predictions would clearly underestimate the experiments. Another handicap of XFEM modelling using the proposed technique is the mesh size dependency of the stresses/strains [50]. P-d curves for XFEM are not presented here, but there show a similar agreement to Figs. 11 and 12, except for small variations on the values of $P_{m}$. Fig. $15(\mathrm{~b})$ shows the values of $P_{m} / P_{\text {mavg, }}$ as defined for Fig. 13(a), for element sizes at the overlap edges (equal length and height) between 0.05 and $0.2 \mathrm{~mm}$, showing that, as expected, this method is extremely mesh dependent.

\section{Concluding remarks}

The main objective of this work was to evaluate the capabilities and/or limitations of using the current implementations of Cohesive Zone Modelling or eXtended Finite Element Modelling available in ABAQUS ${ }^{\boldsymbol{s}}$ to simulate the behaviour and strength of adhesively bonded joints. With this purpose, single- and double-lap 
joints between aluminium adherends were considered, bonded with the brittle adhesive Araldites AV138. A variety of overlap lengths was tested, between 5 and $20 \mathrm{~mm}$, to test both solutions for fracture modelling under different load gradients, i.e., between an approximately even level of shear stresses along the bond up to the large shear stress gradients found in joints with bigger bond lengths. The direct comparisons between the experimental data and the output of the simulations revealed accurate predictions for the Cohesive Zone Modelling technique. This was expected, since this technique has been extensively validated for a wide variety of engineering problems, with positive results being expected, provided that the shape of the chosen cohesive laws are consistent with the constitutive behaviour of the material they are simulating. The eXtended Finite Element Method, expanding Cohesive Zone Modelling by the allowance of crack propagation along arbitrary directions within solid continuum elements, did not show to be suited for damage propagation in bonded joints as it is currently implemented, since the direction of crack growth is ruled by the maximum principal stresses/strains at the crack tip which, in bonded joints, invariably leads to damage growth towards and within the adherends. This clearly does not reflect the behaviour of bonded joints and can be attributed to an algorithm for propagation not still suited to multi-material structures as it does not search for failure points outside the crack tip nor following the interfaces between different materials. Restriction of damage propagation only for the adhesive layer is also rendered unfeasible to surpass this limitation as crack propagation halts when the crack attains the aluminium. Due to the brittleness of the adhesive used, the eXtended Finite Element Method was used to predict failure by damage onset at the overlap edges, which showed satisfactory results in terms of quantitative results and dependence with the overlap length, but extremely mesh dependent. Some principles were proposed to modify this promising technique for the simulation of bonded joints.

\section{Acknowledgements}

The authors would like to thank the Portuguese Foundation for Science and Technology for supporting the work here presented.

\section{References}

[1] Messler RW. Joining of advanced materials. Stoneham, USA: Butterworths/ Heinemann; 1993.

[2] R.D.S.G. Campilho, de Moura MFSF, Domingues JJMS. Modelling single and double-lap repairs on composite materials. Composites Science and Technology 2005;65:1948-58.

[3] Volkersen O. Die nietkraftoerteilung in zubeanspruchten nietverbindungen konstanten loschonquerschnitten. Luftfahrtforschung 1938;15:41-7.

[4] Goland M, Reissner E. The stresses in cemented joints. Journal of Applied Mechanics 1944;66:17-27.

[5] Szépe F. Strength of adhesive-bonded lap joints with respect to change of temperature and fatigue. Experimental Mechanics 1966;6:280-6.

[6] Hart-Smith LJ. Adhesive-bonded single-lap joints. NASA Technical Report CR112236. Hampton, USA: Langley Research Centre; 1973.

[7] Pirvics J. Two dimensional displacement-stress distributions in adhesive bonded composite structures. The Journal of Adhesion 1974;6:207-28.

[8] Panigrahi SK, Pradhan B. Three dimensional failure analysis and damage propagation behavior of adhesively bonded single lap joints in laminated FRP composites. Journal of Reinforced Plastics and Composites 2007;26:183-201.

[9] Wooley GR, Carver DR. Stress concentration factors for bonded lap joint. Journal of Aircraft 1971;8:817-20.

[10] Harris JA, Adams RD. Strength prediction of bonded single-lap joints by nonlinear finite element methods. International Journal of Adhesion \& Adhesives 1984;4:65-78.

[11] Bigwood DA, Crocombe AD. Non-linear adhesive bonded joint design analyses. International Journal of Adhesion \& Adhesives 1990;10:31-41.

[12] Crocombe AD, Adams RD. An elastoplastic investigation of the peel test. The Journal of Adhesion 1982;13:241-67.

[13] Lee SJ, Lee GL. Development of a failure model for the adhesively bonded tubular single lap joint. The Journal of Adhesion 1992:40:1-14.
[14] Qian ZQ, Akisanya AR. An investigation of the stress singularity near the free edge of scarf joints. European Journal of Mechanics A/Solids 1999;18:443-63.

[15] Dragoni E, Mauri P. Intrinsic static strength of friction interfaces augmented with anaerobic adhesives. International Journal of Adhesion \& Adhesives 2000;20:315-21.

[16] Feih S, Shercliff HR. Adhesive and composite failure prediction of single-L joint structures under tensile loading. International Journal of Adhesion \& Adhesives 2005;25:47-59.

[17] Cavalli MN, Thouless MD. The effect of damage nucleation on the toughness of an adhesive joint. The Journal of Adhesion 2001;76:75-92.

[18] Campilho RDSG, de Moura MFSF, Domingues JJMS. Stress and failure analyses of scarf repaired CFRP laminates using a cohesive damage model. Journal of Adhesion Science and Technology 2007;21:855-970.

[19] Cui W, Wisnom MR. A combined stress-based and fracture-mechanics-based model for predicting delamination in composites. Composites 1993;24:467-74.

[20] Petrossian Z, Wisnom MR. Prediction of delamination initiation and growth from discontinuous plies using interface elements. Composites: Part AApplied Science and Manufacturing 1998;29:503-15.

[21] Feraren P, Jensen HM. Cohesive zone modelling of interface fracture near flaws in adhesive joints. Engineering Fracture Mechanics 2004;71:2125-42.

[22] Campilho RDSG, de Moura MFSF, Domingues JJMS. Using a cohesive damage model to predict the tensile behaviour of CFRP single-strap repairs. International Journal of Solids and Structures 2008;45:1497-512.

[23] R.D.S.G. Campilho, de Moura MFSF, Pinto AMG, Morais JJL, Domingues JJMS. Modelling the tensile fracture behaviour of CFRP scarf repairs. Composites: Part B-Engineering 2009;40:149-57.

[24] Kafkalidis MS, Thouless MD. The effects of geometry and material properties on the fracture of single lap-shear joints. International Journal of Solids and Structures 2002;39:4367-83

[25] Belytschko T, Black T. Elastic crack growth in finite elements with minimal remeshing. International Journal of Fracture Mechanics 1999;45:601-20.

[26] Melenk JM, Babuska I. The partition of unity finite element method: basic theory and applications. Seminar fur Angewandte Mathematik, Eidgenossische Technische Hochschule, Research Report No. 96-01, January, CH-8092 Zurich, Switzerland, 1996.

[27] Moës N, Dolbow J, Belytschko T. A finite element method for crack growth without remeshing. International Journal for Numerical Methods in Engineering 1999;46:131-50.

[28] Mohammadi S. Extended finite element method for fracture analysis of structures. New Jersey, USA: Blackwell Publishing; 2008

[29] Sukumar N, Moës N, Moran B, Belytschko T. Extended finite element method for three-dimensional crack modeling. International Journal for Numerical Methods in Engineering 2000;48:1549-70.

[30] Daux C, Moës N, Dolbow J, Sukumark N, Belytschko T. Arbitrary branched and intersecting cracks with the extended finite element method. International Journal for Numerical Methods in Engineering 2000;48:1741-60.

[31] Moës N, Belytschko T. Extended finite element method for cohesive crack growth. Engineering Fracture Mechanics 2002;69:813-33.

[32] Elguedj T, Gravouil A, Combescure A. Appropriate extended functions for XFEM simulation of plastic fracture mechanics. Computer Methods in Applied Mechanics and Engineering 2006;195:501-15.

[33] Dolbow J, Moës N, Belytschko T. An extended finite element method for modeling crack growth with frictional contact. Finite Elements in Analysis and Design 2000;36:235-60.

[34] Khoei AR, Nikbakht M. Contact friction modeling with the extended finite element method (X-FEM). Journal of Materials Processing Technology 2006; $177: 58-62$

[35] Fagerström M, Larsson R. Theory and numerics for finite deformation fracture modelling using strong discontinuities. International Journal for Numerical Methods in Engineering 2006;66:911-48

[36] PMGP Moreira, de Jesus AMP, Ribeiro AS, de Castro PMST. Fatigue crack growth in friction stir welds of 6082-T6 and 6061-T6 aluminium alloys: a comparison. Theoretical and Applied Fracture Mechanics 2008;50:81-91.

[37] ASTM-E8M-04. Standard test methods for tension testing of metallic materials [Metric], 2004.

[38] NF T 76-142. Méthode de preparation de plaques d'adhésifs structuraux pour la réalisation d'éprouvettes d'essai de caractérisation, 1988.

[39] ISO 11003-2:1993(E). Adhesives-determination of shear behavior of structural bonds, Part II: thick adherend tensile test method, 1993.

[40] da Silva LFM, RAM da Silva, JAG Chousal, AMG Pinto. Alternative methods to measure the adhesive shear displacement in the thick adherend shear test. Journal of Adhesion Science and Technology 2008;22:15-29.

[41] Goyal VK, Johnson ER, Goyal VK. Predictive strength-fracture model for composite bonded joints. Composite Structures 2008;82:434-46.

[42] Radice JJ, Vinson JR. On the analysis of adhesively bonded structures: a high order semielastic adhesive layer model. Composites Science and Technology 2008;68:376-86

[43] ABAQUS ${ }^{\text {s }}$ HTML Documentation, Dassault Systemes, 2009.

[44] Alfano G. On the influence of the shape of the interface law on the application of cohesive-zone models. Composites Science and Technology 2006;66:723-30.

[45] Sukumar N, Prevost JH. Modeling quasi-static crack growth with the extended finite element method, Part I: computer implementation. International Journal for Solids and Structures 2003;40:7513-37.

[46] Banea MD, da Silva LFM. The effect of temperature on the mechanical properties of adhesives for the automotive industry. Proceedings of the IMechE, Part L: Journal of Materials: Design and Applications 2010;224:51-62. 
[47] Banea MD, da Silva LFM. Mechanical characterization of flexible adhesives. The Journal of Adhesion 2009;85:261-85.

[48] Campilho RDSG, de Moura MFSF, Domingues JJMS. Numerical prediction on the tensile residual strength of repaired CFRP under different geometric changes. International Journal of Adhesion \& Adhesives 2009;29:195-205.
[49] Xie D, Waas AM. Discrete cohesive zone model for mixed-mode fracture using finite element analysis. Engineering Fracture Mechanics 2006;73:1783-96.

[50] Panigrahi SK, Pradhan B. Three dimensional failure analysis and damage propagation behavior of adhesively bonded single lap joints in laminated FRP composites. Journal of Reinforced Plastics and Composites 2007;26:183-201. 